\title{
Investigating the Effect of Copper Oxides and Alumina Nanoparticles on Enhanced Oil Recovery in Carbonate Reservoirs
}

\author{
Mohamed Waleed ${ }^{1}$, Sayed Gomaa ${ }^{1,2^{*}}$, Samir Khaled ${ }^{1,2}$, Ramadan Emara ${ }^{1,2}$, Atef Abdelhady ${ }^{1}$, \\ Mohamed Elwageeh ${ }^{1}$ \\ ${ }^{1}$ Petroleum Engineering and Gas Technology Department, Faculty of Engineering, The British University in \\ Egypt, El Sherouk City, Cairo, Egypt. \\ ${ }^{2}$ Mining and Petroleum Engineering Department, Faculty of Engineering, Al-Azhar University, Cairo, Egypt \\ *Corresponding Author: Sayed Gomaa, Petroleum Engineering and Gas Technology Department, Faculty \\ of Engineering, The British University in Egypt, El Sherouk City, Cairo, Egypt.
}

\begin{abstract}
In this paper, eight limestone core samples will be used in this experiment and the flooding process will be done by using a core flood system. The displacing fluid will be prepared by using solutions of Copper Oxide and Alumina nanoparticles at concentrations of (0.0125, 0.025, 0.07, and $0.5 \mathrm{wt} \%)$ and (0.01, 0.05, 0.1, and $0.5 \mathrm{wt} \%$ ) respectively, then it will be used in flooding the core samples containing crude oil with viscosity of $3.25 \mathrm{cp}$. The sizes of used nanoparticles are from 30 to $50 \mathrm{~nm}$ for Copper Oxide and 5 for Alumina. Each case of Nano flooding will be compared with the basic flooding of water injection with a salinity of 50,000 ppm. This work indicates that Copper Oxide and Alumina nanoparticles at low concentrations have the best results more than high concentrations. Copper Oxide at a concentration of $0.0125 \mathrm{wt} \%$ has a large ability to change from formation wettability to be more water wet. Alumina nanoparticle at concentration of 0.01 wt $\%$ and Copper Oxide nanoparticle at concentration of $0.0125 \%$ have the best results in increasing the oil recovery factor by percentage of $24 \%$
\end{abstract}

Keywords: Nano - Copper - Alumina - Flooding - Carbonate Reservoir - Enhanced

\section{INTRODUCTION}

The process of enhanced oil recovery shows a huge result in recovered trapped stranded oil at the pores of the reservoir after applied the recovery methods of primary and secondary techniques by using techniques of driven snap-off and capillary pressure. These techniques recover two-third of the original oil in place, methods of EOR have a big impact on oil production as increasing only $1 \%$ in recovery factor can produce about 70 billion barrels from conventional oil reserve. EOR uses lots of technologies and processes that working on increasing the recovery factor of oil from the existing reservoirs. These processes contain injection of fluids and recently injection of microbes to support the natural energy of the reservoir and make an effective displacement of oil into the production well, as a result of this injection there are interactions occurred between the fluids of injection and rocks/oil of the reservoir, these interactions make a favorable conditions for oil production [1-3]. Nanotechnology is one of the most important discoveries that have occurred in the field of industry, especially in the oil and gas industry, because this technology has revolutionized many innovations related to aspects of the oil and gas industry. The material of the Nano is characterized by having high concentrated volume and large surface area. These dimensional effects have given them distinct magnetic, mechanical, thermal, and chemical properties. The nanomaterials are also distinguished as they are treated as a chemical treatment. Their properties can be modified for a specific technical purpose [4-7].

\subsection{Application of Nanotechnology in the Oil and Gas Industry}

Since the discovery of nanotechnology in the 1980s, and it has had a major impact on all disciplines of biology, chemistry, physics, mechanics, and material balance to provide a new platform for solving complex problems. The nanomaterials are described as crystals, sheets, rods, and particles with size ranging from 1 to $100 \mathrm{~nm}$, and it provides practical application in lots of industrial sectors, as we will see in this research their impact on the oil and gas industry. After the development of nanotechnology, 
it becomes reliable in the oil and gas industry to solve the complex problem in the oil field and chiefly to improve oil recovery, the trend towards the use of nanotechnology has become significant, especially in the last 5 years. Several theoretical and scientific studies have been created to develop these industries. And now we will study the effect of this technology on exploration, drilling, and extraction [8-10].

Applications of nanotechnology are applied widely in many regions of oil and gas development and exploration, And nanotechnology has surpassed many other technologies that are used in exploration, so it is constantly being developed to increase oil and gas sources and it will be applied developed to the following aspects. Nno-characterization and numerical simulation technique provides an accurate reference in the analysis and description of the reservoir and helps greatly in decision-making $[6,11]$. Nano-sensing technology is a black box decoding of the oil reservoir. Nano-robot has extremely accurate sensors is the best way to detect where the oil is and how it is spreading [12,13]. Nanomaterials have lots of applications on nanomaterial as it works as catalysts to facilitate the exploration, drilling and extraction process [14-16].

Nanotechnology has a significant impact on the process of drilling and completion, as it helps in solving the complex problem, especially the problem that related to formation and environment. There are some applications of using nanotechnologies and nanomaterial in drilling and completion [15]. Adding nanomaterials into drilling fluids helps in stabilizing the wellbore as particles of Nano form filter cake on the wellbore to reduce from the wellbore collapse and water swilling, also it helps in reduce filter loss as a nanoparticle works on seal pores of the wellbore to reduce fluid losses, helps in improving rheology by increase viscosity and shearing force of the system, and finally increase the thermal stability of drilling system [17-20]. Nanocomposite helps in develop equipment of drilling to be lighter and more reliable and reduces fluid invasion into shales [21,22]. Nano coating helps in resist corrosion of drilling equipment, higher quality of drilling component and make it stronger [23-25].

The idea of this technology is to add nanomaterial into based water fluid to generate Nano-fluids, it can be used with and without chemicals to improve from oil recovery. There are lots of papers and researches that are published about this mechanism and the results seem very interesting. Lots of published papers, the results of experimental works indicate the possibility of increasing and improve the recovery of oil by using Nano-fluids. It also noticed that the percentage of EOR is depending on some main properties of Nano-fluids such as (types of material, percentage of concentration, and size of the particle). According to there are lots of different mechanisms that can be applied in EOR by using Nano-fluids. These mechanisms are: (reduction in interfacial tension, increase in the viscosity of the aqueous solution, wettability alteration, disjoining pressure changes, slug-like displacement, decrease viscosity of the oil, log jamming, and combination between different mechanisms). The next slide will analyze each of these mechanisms to define how it works and the expected problems.

\subsection{Mechanisms of Using Nano-Fluids in Wettability Alteration}

Wettability [26-28] has a significant impact on the percentage of oil recovery. Some nanomaterials can change the wettability of oil-rock-water systems by buildup wedge film structure in contact of three phases. Nanoparticles create a wetting wedge between oil bubbles and solid surfaces to help in separate oil bubbles from the surface and change the wettability of the system to be water wet, as shown in Figure 1.

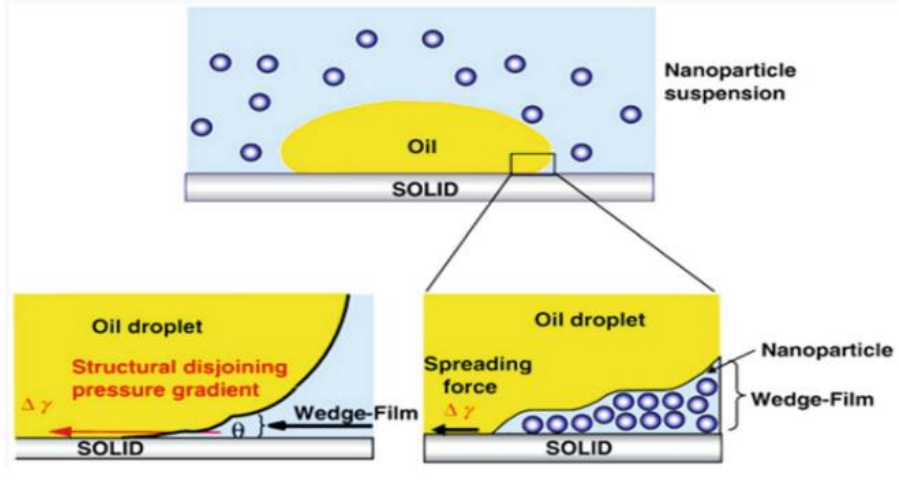

Figure1. Nanoparticles creates wedge-film structure 
The angle of contact between $n$-heptane and water on calcite clean plates in oil was 180 degrees. By using zirconium oxide nanoparticle (Zio2) with concentration $(0.05 \%$, and $0.10 \%$ ) and size $24 \mathrm{~nm}$ this angle between $n$-heptane and water is changed according to the type of concentration used in treatment from 180 to 32 degree to change the wettability of rock from oil-wet to strongly water-wet. And the experiment mentioned that angles between $n$-heptane and water reduce with increasing aging time and to achieve the best alteration aging time should not be less than 48hr [29].

\subsection{Applications of Nano-Fluids to Reduce Interfacial Tension}

The reduction of interfacial tension between oil and water is considered one of the important factors that affect the efficiency of oil recovery. Lots of papers are mentioned that using Nano-fluids in EOR shows good results in reduced interfacial tension. The effect of nanoparticles and Nio/Sio2 nanocomposites to reduce the interfacial tension and surface tension (S.T) for a sample of heavy oil produced from Soroush field located in Iran with $1310 \mathrm{cP}$ of viscosity, $0.965 \mathrm{~g} / \mathrm{cm} 3$ of density was investigated by some researchers the interfacial tension between oil/water without nanoparticles was $29.02 \mathrm{mN} / \mathrm{m}$ at $22{ }^{\circ} \mathrm{C}$, the experiment was done by injecting Nano-fluid water-based of $30 \mathrm{wt} \%$ Sio2/Nio and $28 \mathrm{wt} \% \mathrm{Fe} @$ Sio2/Nio. The results of the experiment are decreased in IFT to $1.28 \mathrm{mN} / \mathrm{m}$ and less than $1 \mathrm{mN} / \mathrm{m}$ and with repeat the experiment by addition of concentration, salinity and aging time they found that the pest concentration to reduce the IFT and S.T is $30 \mathrm{wt} \%$ of Sio2/Nio in diluted water $[10,26]$.

\subsection{Applications of Nano-Fluids to Increase the Viscosity of the Aqueous Solution}

Increasing viscosity of the aqueous solution is a very important factor in EOR, as it plays an important role in increasing the efficiency of oil displacement and oil swilling. Many papers made researches about how to increase the viscosity of water by using nanoparticles. According to [30], nanoparticles could increase the viscosity of aqueous solution with $100 \%$, for example, the viscosity of sulphonate solution increase from $1 \mathrm{cp}$ to $2 \mathrm{cp}$ by adding $0.01 \mathrm{wt} \%$ of nanoparticles. , Verag mentioned that nanoparticles can be used to improve interventions of water-flooding to different strong permeability zones that effect on displacement of oil, by adding capsules of nanomaterial during injection to increase the viscosity of water [31].

\subsection{Applications of Nano-Fluids to Decrease Oil Viscosity at Reservoir Conditions}

Oil viscosity is one of the main factors that affect oil recovery, so many petroleum institutes are researched about how to apply nanotechnology to reduce the viscosity of heavy oil. The next slide will show some of these researches and experiments.

According to [32], they worked on research about reducing the viscosity of heavy oil by using nanoparticles. The research involved two different experiments, the first experiment depends on inject surfactant-based fluid with nanoparticles into the oil sample, and the second one depends on examined the effect of Copper Oxide nanoparticle without surfactant-based fluid. Copper Oxide (Cuo) nanoparticles are selected due to their easy availability, low cost, and high thermal conductivity. The experiment is done on two different samples of heavy oil with different viscosities produced from wells located in Oklahoma, USA. Oil samples are mixed with an emulsion of Copper Oxide nanoparticles at different concentrations with 50nm particle size. The results of this experiment refer to $0.1 \mathrm{wt} \%$ of Cuo nanoparticles with size $50 \mathrm{~nm}$ give the best reduction in oil viscosity and it is expected to give a more positive result with using Cuo nanoparticles with concentration $0.02 \mathrm{wt} \%$.

According to [33], to solve the problems of reservoirs by using nanotechnology, they study the effect of some different materials of nanoparticles on oil reservoirs. These experiments are depending on two conducted sets. the first one is displacing the oil with injected nanofluid and the second one is to soak the sand in nanofluid for 60 days before starting displacement process.

This experiment is done by an oil sample with $0.9114 \mathrm{~g} / \mathrm{cc}$ of density, $53.27735 \mathrm{cp}$ of viscosity, and 22.44 API. The result of this experiment shows:

- Nanoparticles of aluminum oxide improve oil recovery by reducing oil viscosity.

- Saline and hydrophobic silicon oxide dispersed in ethanol improve oil recovery by change rock wettability. 
- Ethanol without nanoparticle enhances oil recovery by reducing interfacial tension.

- Types of dispersion fluids very important factors to improve oil recovery.

- Magnesium and zinc oxide make permeability problems and show low recovery results.

\section{EXPERIMENTAL WORK}

As mentioned before, the main purpose of this paper is to find the best way to improve the oil recovery factor, and this is done by changing the properties of crude oil by applying different types of nanomaterials at different concentrations on crude oil. These nanomaterials will be applied by using the strategy of nano flooding techniques, this strategy is mentioned in lots of certain researches and it works on improving the technique of base water flood. As nanomaterials help in improve the properties of crude oil such as reducing the oil viscosity, reducing the interfacial tension, changing wettability, and...else.

\subsection{Methodology}

In the process of investigating the effect of nano particles on enhanced oil recovery (EOR), usually, the actual limestone cores of the reservoir are used in lab experiments to give the optimum evaluation of the effects of different displacing media and reservoir physical properties. Usually, there are three different sizes of samples in the laboratory: $3.8 \mathrm{~cm}$ core diameter, $2.8 \mathrm{~cm}$ core diameter, and full core diameter.

\subsection{Core Flood System}

A core flood system is used to perform the flooding experiments. These experiments are helping in discover the mechanism of various flooding operations, improving the displacement efficiency, and providing a theoretical basis for oil industry development applications.

The main technical parameters of the experimental flooding system with full diameter core are sample length, sample diameter, confining pressure, temperature, and displacement pressure.

The experimental flooding system with a full diameter core consists mainly of a full-diameter core holding system, a displacement system, and a metering system. The schematic diagram for the system is shown in Figure 2

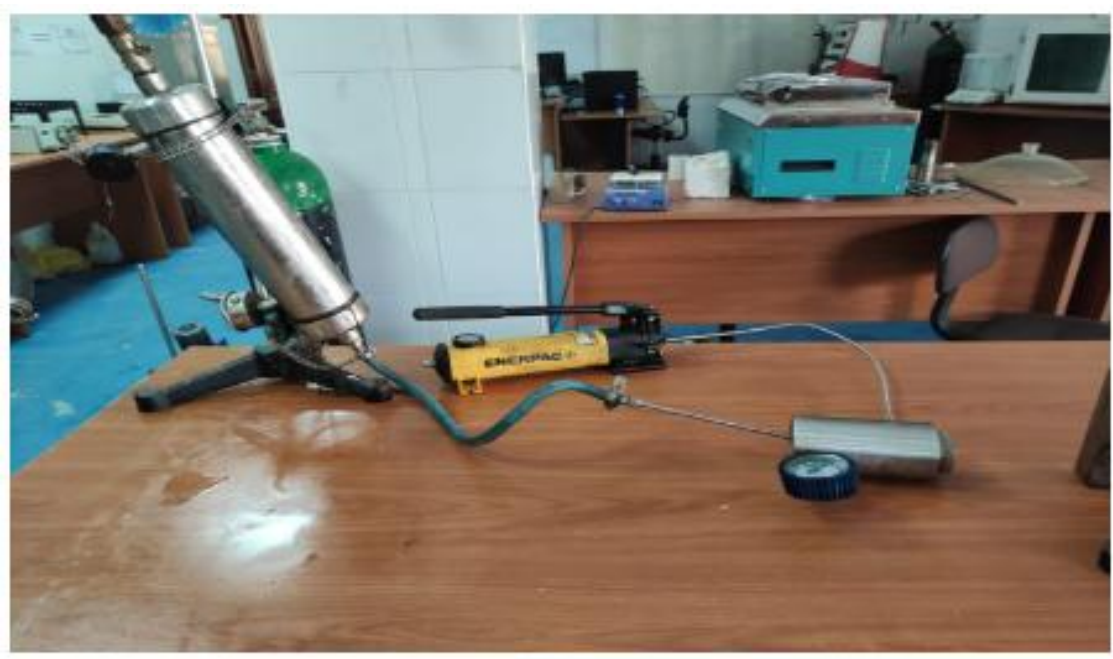

Figure2. Core holder displacement system

The core holding system is mainly consisting of a core holding cavity and a flip bracket. The core holding cavity is a bore designed to accommodate the core, it mainly consists of regulating plugs, sleeves of rubber, and cavity. The sleeves of rubber are in the cavity, and the core is in the sleeves of rubber. The sleeve diameter is slightly greater than the core diameter. When applied with confining pressure to the core holder, the core can be fully tightened to ensure that the displacing medium passes through the core. The system of the core holding consists of a bracket where the holder's cavity can be flipped through. The main reason for the flip bracket is designed for cores with large diameters to make sure that the core will be fully displaced and fully saturated during the experiment. 
The system of displacement consists of two kinds of displacement functions of liquid flooding and gas flooding. The system of gas flooding includes a nitrogen gas cylinder with high pressure, a relief valve, and a sensor for pressure. The gas is inserted from the high-pressure gas cylinder, and the required displacement pressure is controlled through the pressure relief valve. The process of displacement is work by press different media into the system of core holding by a pump. The flow rate and the pressure are determined by the requirement of each experiment. After the liquid passing through the core, the amount of fluid is measured at the outlet.

\subsection{Sample Preparation in the Laboratory}

The chosen samples for laboratory experiments are taken from limestone rock. Ten limestone cores are selected for cutting and grinding to make sure that each core has a cylindrical shape and the two ends are flush. According to standard of petroleum industry the cores must be washed then put in oven to be fully dried and usable

\subsection{Petrophysical Properties for Each Limestone Core Sample}

The core samples are dried and the length, the diameter and the dry weight are measured for each core sample and then the bulk volume is calculated. By using sodium chloride for preparing the brine with a concentration of 50,000 ppm, then this brine is used on the process of initiating the core sample to make it fully saturated with this brine. After making sure that the core became fully saturated with brine, measure the saturated weight of the core sample. By using the saturated weight and the dry weight of core, the pore volume is calculated and then the porosity is calculated.

To calculate the absolute permeability of the core, the core sample is put inside the core holder and the water is injected into the core sample by using the same brine water for 1 minute and using constant pressure, after 1 minute measure the displaced water. Now calculate the absolute permeability (K) of core sample by using this equation:

$$
k=\frac{q_{w} \times \mu_{w} \times L}{\frac{\pi}{4} d^{2} \times \frac{\Delta p}{14.7}} \times 1000
$$

\subsection{Core Sample Initiation}

After finishing the first run, put crude oil in displacement tank (the viscosity of crude oil is $3.25 \mathrm{cp}$ ) to make oil displaces the brine water, this step is very important to simulate the initial conditions of the reservoir. The volume of displaced brine water equal to volume of crude oil enters to the core sample. to calculate the connate water of core sample, subtract the amount of oil enter to core sample from the total amount of water that injected initially to saturate the core sample. Now the core sample is having the same condition of reservoir and ready for the flooding.

\subsection{Procedures of the Experiment}

- Clean the core samples and put it in the oven to be fully dried

- Measure the dry weight of each core, then measure the length and the diameter of each core.

- Put the cores in the prepared brine water and put them with brine in a vacuum to be fully saturated with brine water. Measure the saturated weight of each core sample.

- Calculate the pore volume of each core by subtracting the saturated weight from dry weight and divide the result on the density of brine water

- Simulate the core sample with the reservoir, by injecting crude oil into the core to displace the brine water. Not all amount of water is displaced, the remaining water called connate water. The amount of oil entered the core is the same amount of water displaced from the core.

- Inject displaced fluid into the core to displace crude oil. Measure the amount of oil recovery and recorded the time with each fraction of injected pore volume, to determine the flow rate and the effective permeability.

- Determine the oil recovery factor and the change in wettability. 
Investigating the Effect of Copper Oxides and Alumina Nanoparticles on Enhanced Oil Recovery in Carbonate Reservoirs

\section{RESUlTS ANALYSIS AND DISCUSSIONS}

\subsection{Water Flooding Scenario}

This run considers the base run case, as it represents the conventional scenario of water flooding. In this scenario, the brine water is injected into the core sample to displace the oil. The injected brine water is divided into phases, these phases are $(0.2,0.4,0.6,0.8$. 1. 1.2 1.4, 1.6) of the pore volume. After completing the brine injection, determine the amount of oil that is displaced at each stage to calculate the recovery factor, then construct relative permeability curve to determine if the formation is oil-wet or water-wet. This scenario is a reference scenario for each following case.

\subsection{Nano-Fluid Scenarios}

After constructing the reference scenario (conventional water flooding), the solutions of Copper Oxide and Alumina nanoparticles are prepared to start the nanofluid flooding. The solutions of Copper Oxide and Alumina are injected into the core samples at 4 different concentrations of $(0.0125,0.025,0.07$, and $0.5 \mathrm{wt} \%)$ for Copper Oxide and at concentrations of $(0.0,0.05,0.1$, and $0.5 \mathrm{wt} \%)$ for Alumina. The flooding scenarios are done at 8 phases which are $(0.2,0.4,0.6,0.8,1,1.2,1.4,1.6)$ of pore volume. After completing each run, the oil recovery factor is calculated, and the relative permeability curve and fractional flow curve are constructed. Then each run is compared with the base run of the waterflooding case and the changes at each run and the effect of these changes on oil recovery are determined.

\subsection{Effect of Copper Oxide and Alumina Nanoparticles on Wettability Alteration}

It is founded that the Copper Oxide and Alumina nanoparticles have a clear impact on wettability alteration, displacement efficiency and hence the oil recovery factor. The concentration of Copper Oxide and Alumina nanomaterials play an important role in wettability alternation as shown in Figure 3. All wettability alternation values are positive and these changes in wettability indicate the rock becomes more water wet than conventional waterflooding scenario. Copper Oxide at concentrations of 0.07 and $0.025 \mathrm{wt} \%$, and Alumina at concentration of $0.01 \mathrm{wt} \%$ have a great impact on changing the wettability of rock to be more water wet. But as shown in Figure 3, the better nanomaterial that made the highest changes in wettability of rock to be strongly water-wet is copper at the lowest concentration of $0.0125 \mathrm{wt} \%$.

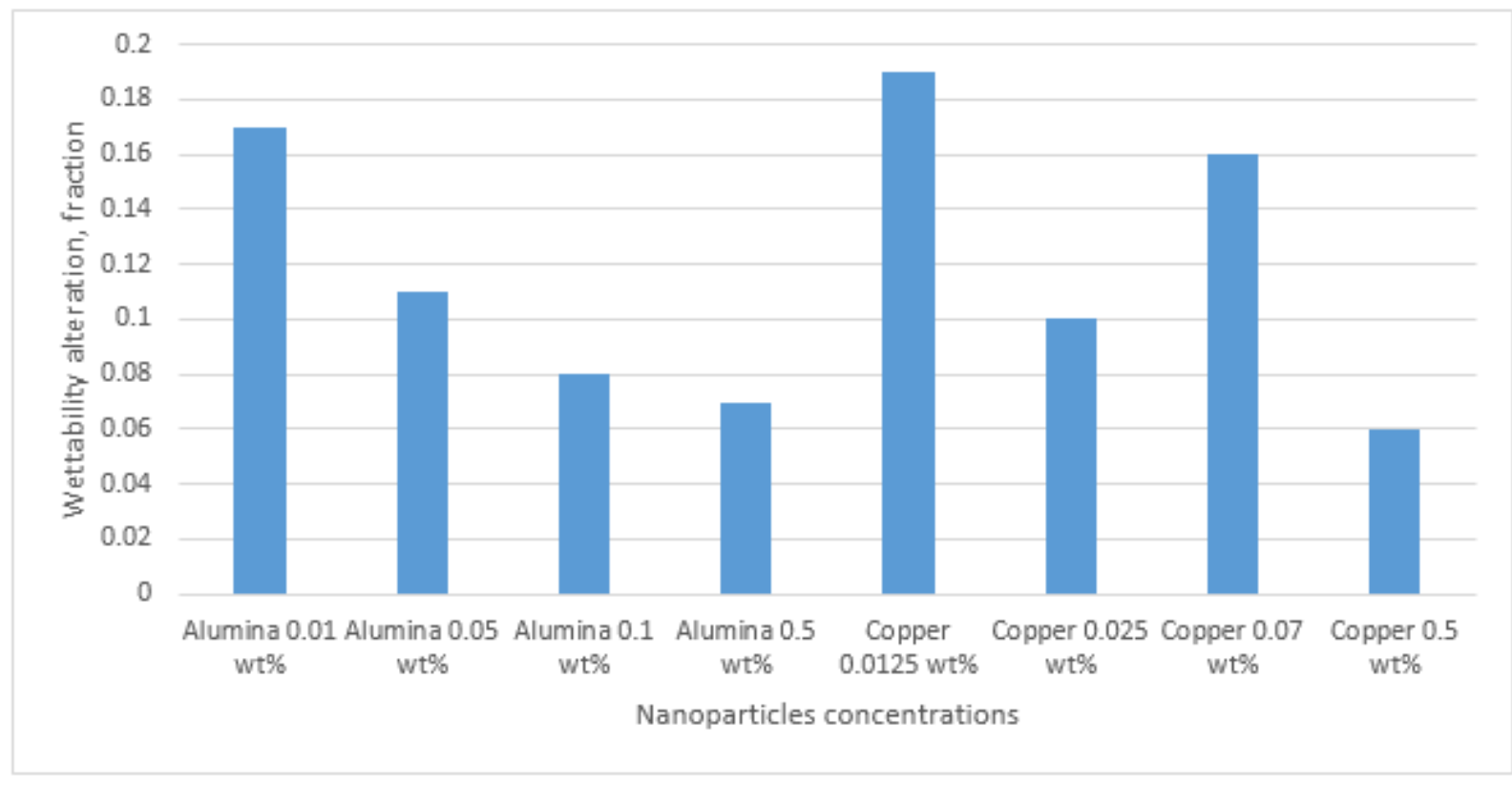

Figure3. Percentage of rock wettability alteration

After completing the Alumina flooding run at a concentration of $0.01 \%$, the oil and water relative permeability curves are drawn. The intersection point of the two curves lies at water saturation of $69 \%$. The results of relative permeability curve for the waterflooding case are compared with the results of the $0.01 \mathrm{wt} \%$ Alumina flooding case. It is noticed that the wettability of reservoir is changed by $17 \%$ to be more water wet as shown in Figure4. 
Investigating the Effect of Copper Oxides and Alumina Nanoparticles on Enhanced Oil Recovery in Carbonate Reservoirs

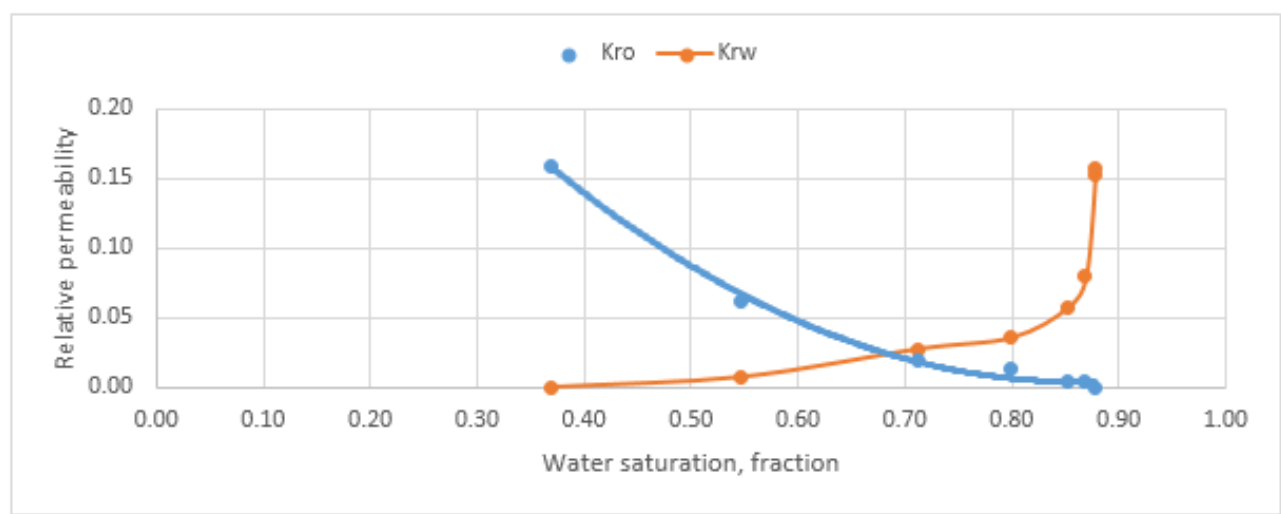

Figure4. Relative permeability curve for 0.01 wt\% Alumina flooding

Also, the effect of Copper Oxide nanopartices at a concentration of $0.0125 \mathrm{wt} \%$ on changing the wettability of formation is very clear, as the intersection point of the two curves changes from $61 \%$ for waterflooding scenario to $80 \%$ for $0.0125 \mathrm{wt} \%$ Copper Oxide flooding case and this indicates the wettability of formation becomes strongly water wet as depicted in Figure 5

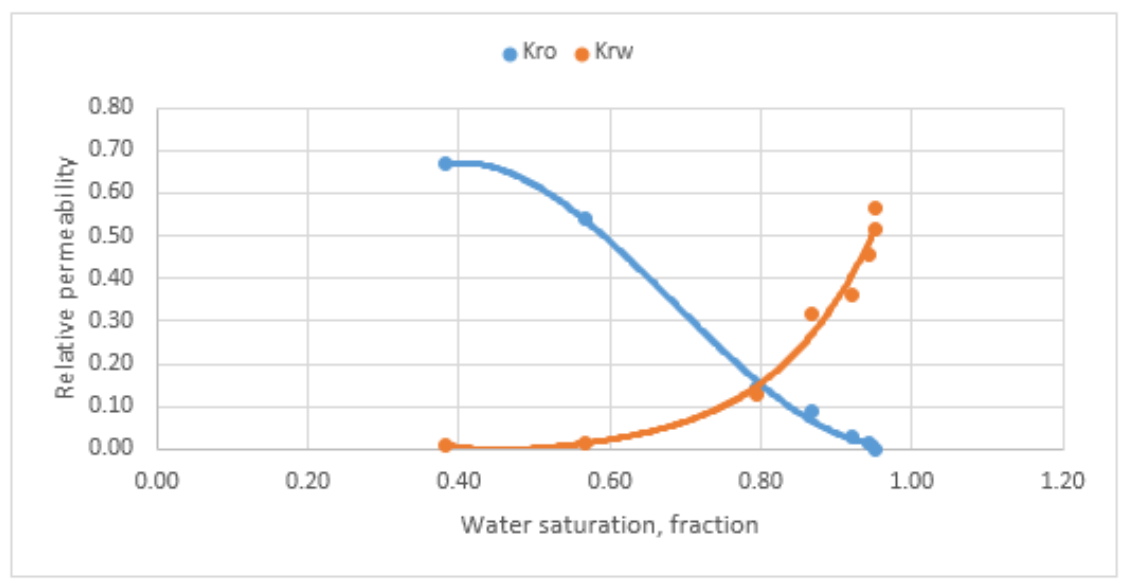

Figure5. Relative permeability curves at 0.0125 wt\% Copper Oxide

\subsection{Effect of Copper Oxide and Alumina Nanoparticles on Oil Recovery}

Figure 6 depicts that nanofluid has a good effect on enhanced oil recovery, but with a difference in the degree of enhancement according to the type of nanomaterial and its ability to enhance the reservoir rock and fluid properties and according to the degree of the concentration. As shown from this figure, nanofluid with low concentration has more effect on enhanced oil recovery than nanofluid with high concentration and this may due to the nanoparticles of high concentration may plug the formation and reduce the oil production.

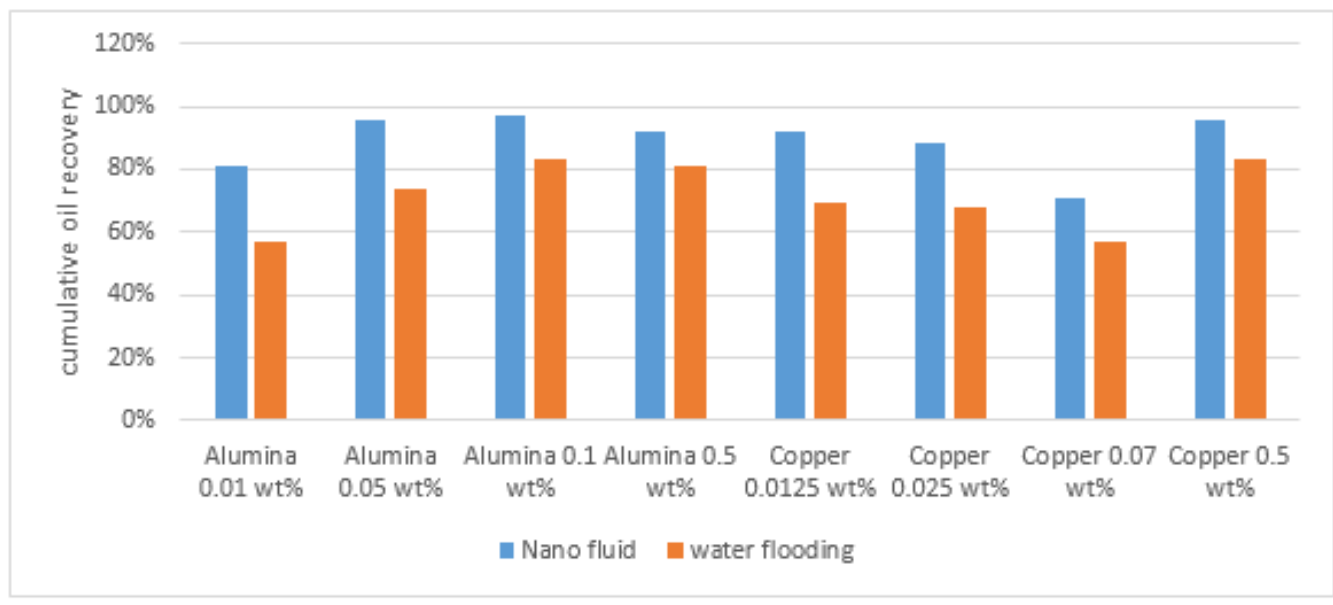

Figure6. Nano flooding vs. water flooding 
Investigating the Effect of Copper Oxides and Alumina Nanoparticles on Enhanced Oil Recovery in Carbonate Reservoirs

Figure 7 shows the flooding effect using Copper Oxide nanofluid at a concentration of $0.0125 \mathrm{wt}$ \%. The incremental increase in the ultimate recovery factor reached up to $23 \%$ of the original oil in place more than that was obtained using the waterflooding

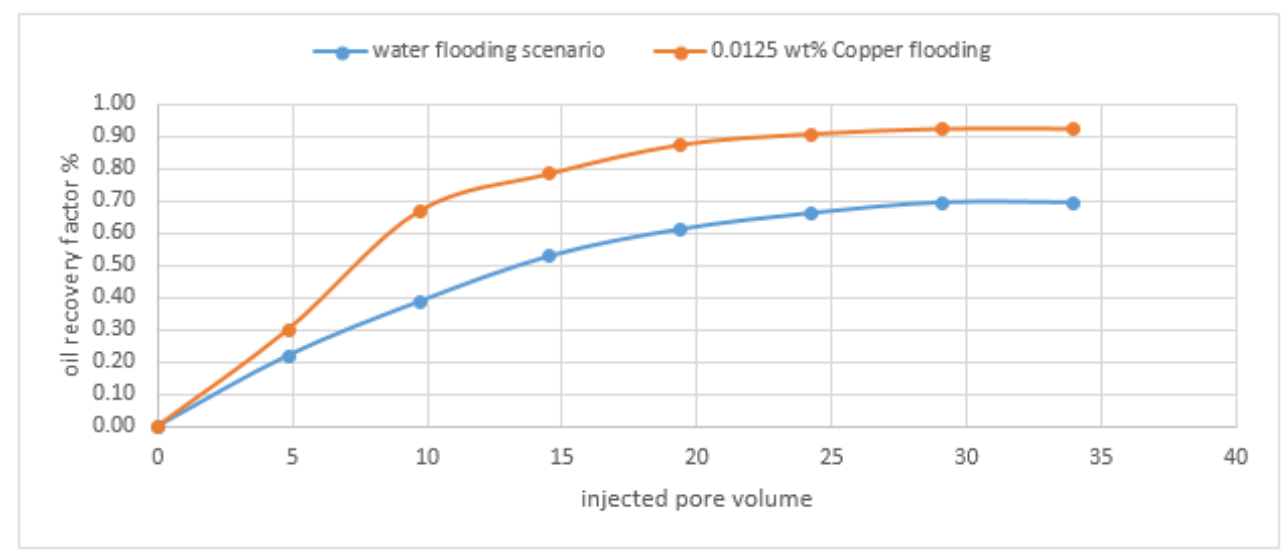

Figure7. Effect of water flooding and 0.0125 wt\% Copper Oxide on oil recovery

Figure 8 shows the flooding effect using Alumina nanofluid at a concentration of 0.01 wt. \%. The incremental increase in the ultimate recovery factor reached up to $24 \%$ of the original oil in place more than that was obtained using the waterflooding

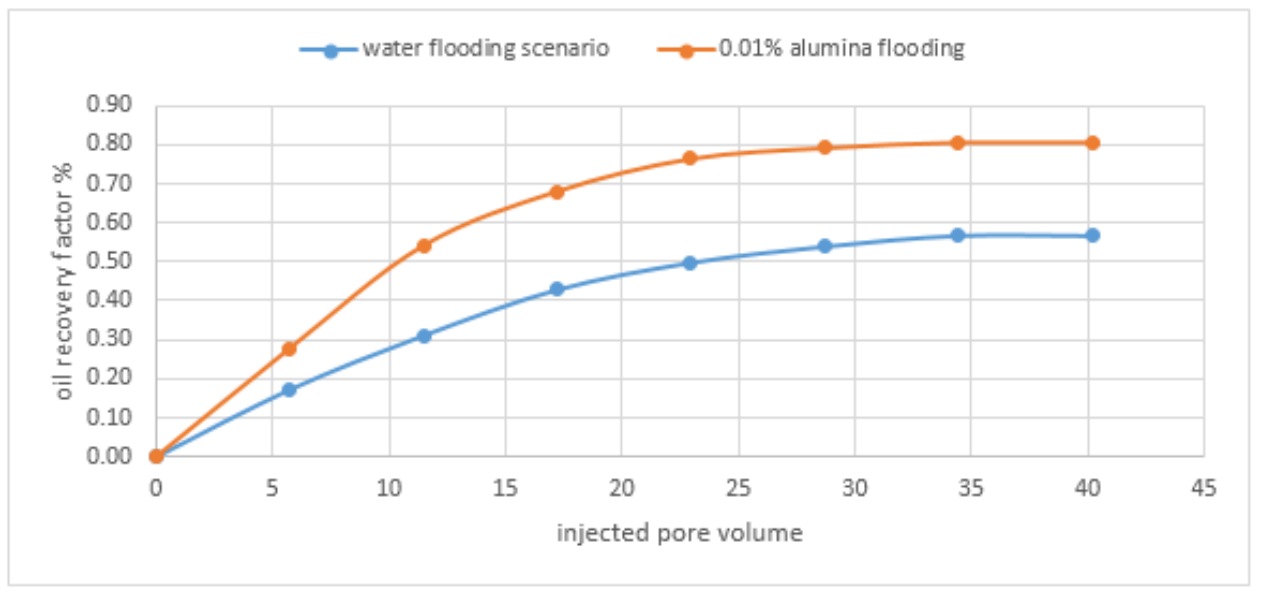

Figure8. Effect of water flooding scenario and 0.01 wt\% Alumina on oil recovery

Figure 9 depicts that the effect of flooding of the core samples using Alumina and Copper Oxide nanoparticles at different concentrations. Alumina flooding at a concentration $0.01 \mathrm{wt} \%$ and Copper Oxide flooding at concentration $0.0125 \mathrm{wt} \%$ gives the highest enhanced oil recovery factors which reached 24\% and 23\% more than water flooding for Alumina and Copper Oxide respectively.

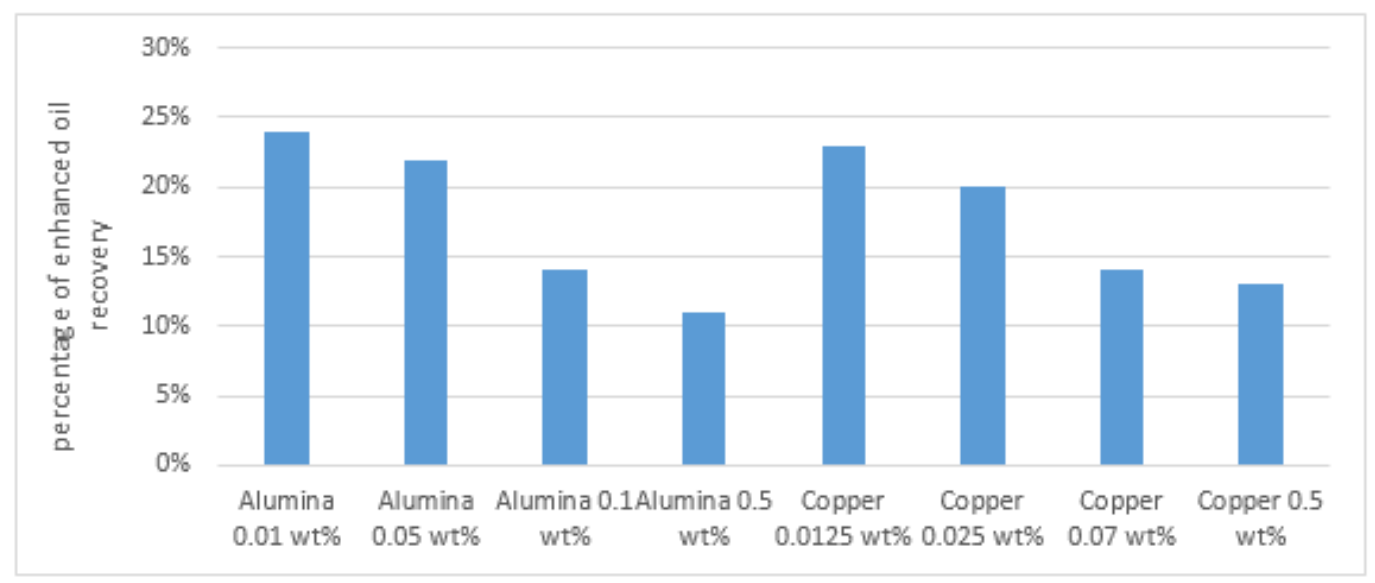

Figure9. Incremental increase in oil recovery 


\subsection{Effect of Copper Oxide and Alumina Nanoparticles on the Displacement Efficiency}

Figures 10 shows the different concentrations of Copper Oxide and Alumina nanoparticles on the displacement efficiency. Generally, flooding with these two nanoparticles increases the displacement efficiency. Copper Oxide flooding at concentration of $0.0125 \mathrm{wt} \%$ increases the displacement efficiency up to $88 \%$. For the scenario of $0.0125 \mathrm{wt} \%$, Copper Oxide flooding, the displacement efficiency was enhanced by $36 \%$ and for the scenario of $0.01 \mathrm{wt} \%$ Alumina flooding, the displacement efficiency was enhanced by $29 \%$ as shown in Figure 11.

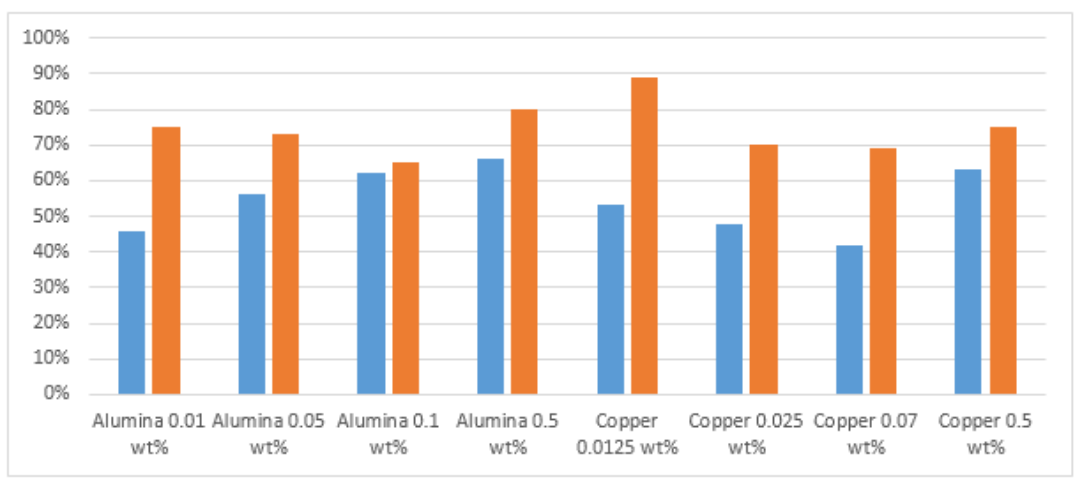

Figure10. Displacement efficiency

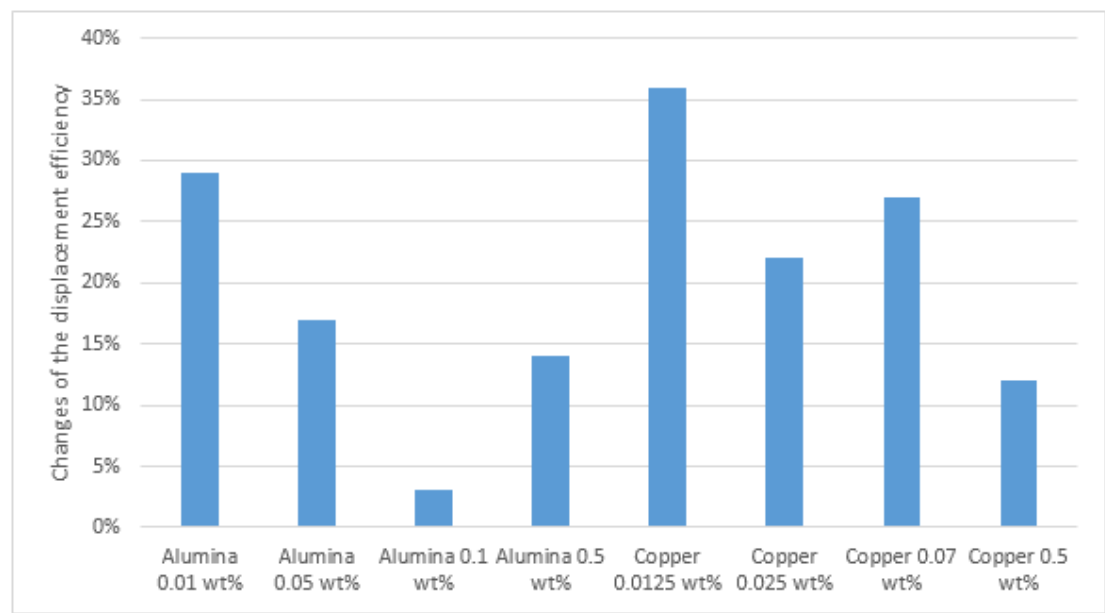

Figure11. Changes in the displacement efficiency

\subsection{Effect of Copper Oxide and Alumina Nanoparticles on Water Breakthrough}

Figure 12 shows the effect of different concentrations of Copper Oxide and Alumina nanoparticles on the water saturation values at breakthrough. The orange color indicates the nano flooding cases and the blue color indicates the waterflooding scenario. As shown from this graph, using these two nanomaterials delays the water breakthrough and the water saturation for example reaches more than $80 \%$ for $0.0125 \mathrm{wt} \%$ Alumina case.

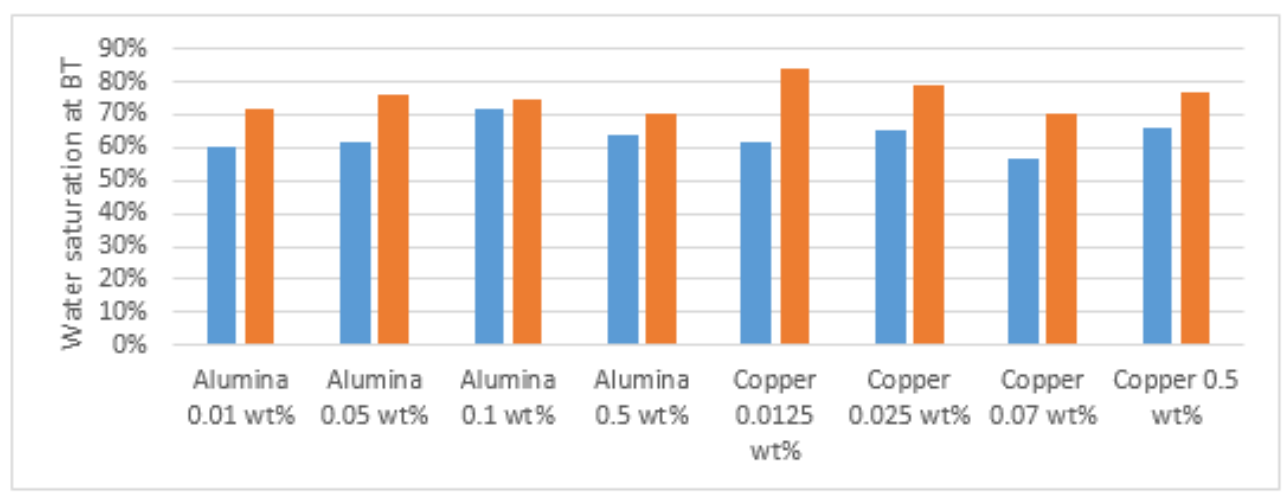

Figure12. Water breakthrough 


\subsection{Effect of Copper Oxide and Alumina Nanoparticles on Water Cut}

Figures 13 and 14 show the fractional flow curve after flooding with Alumina and Copper Oxide nanoparticles at concentration of $0.01 \%$ and 0.0125 respectively. and. From this graph, the of and Alumina nanoparticles on the water cut. Fractional flow curve for Alumina flooding case show that the water saturation at the front is $72 \%$ and the average water saturation behind the front is $840 \%$. Fractional flow curve for Copper Oxide flooding case show that the water saturation at the front is 86 $\%$ and the average water saturation behind the front is $93 \%$.

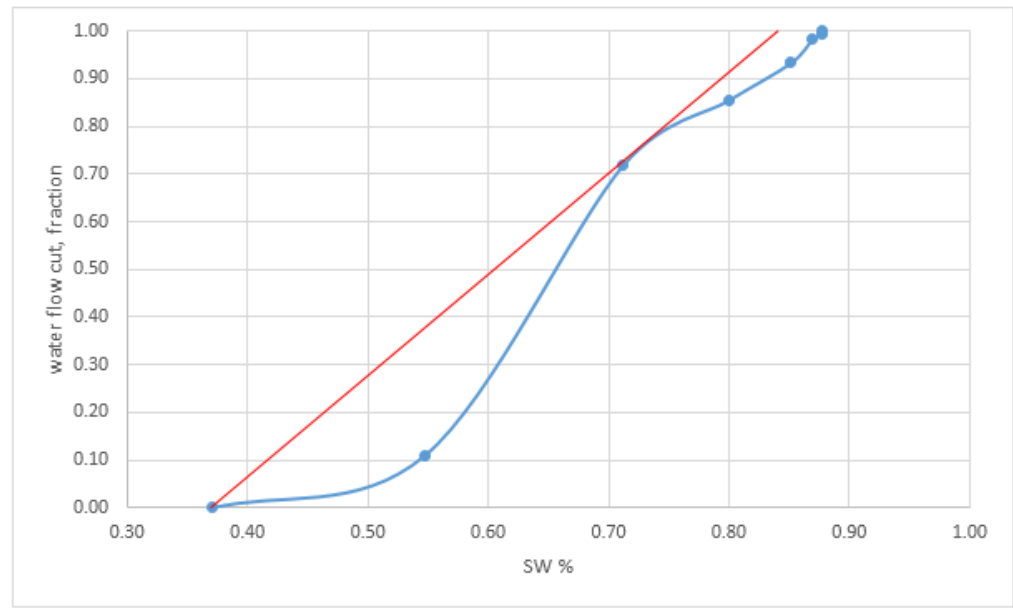

Figure13. Fractional flow curve for 0.01 Alumina flooding

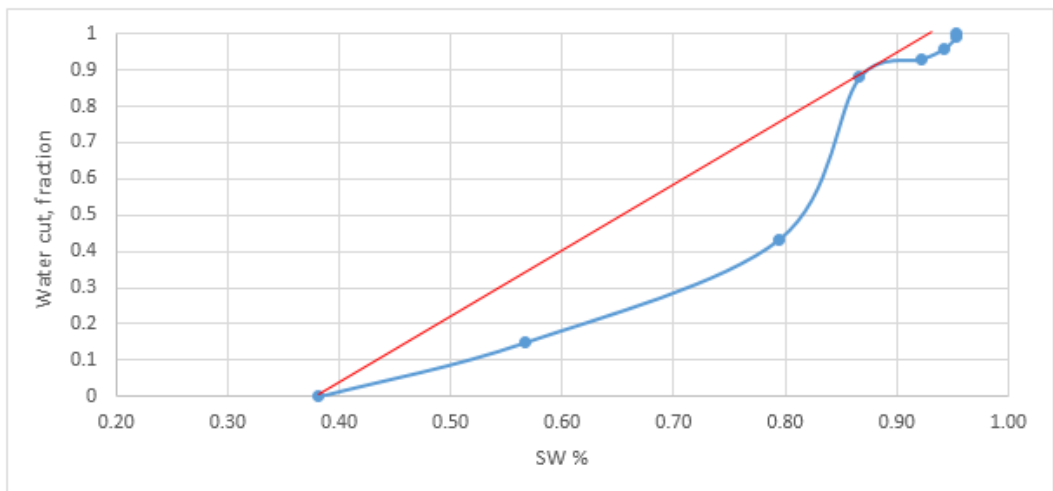

Figure14. Fractional flow curve of 0.0125 wt\% copper

\section{CONCLuSion}

From this paper the following conclusion can be drawn:

- Two types of nanomaterials at four different concentrations are used in this paper to investigate their effects on enhanced oil recovery. These materials are Alumina nanoparticle and Copper Oxide nanoparticle at concentrations of $(0.01,0.05,0.1$, and 0.5$) \mathrm{wt} \%,(0.0125,0.025,0.07$, and 0.5$) \mathrm{wt}$ $\%$ respectively.

- Alumina nanoparticle at concentration of $0.01 \mathrm{wt} \%$ and Copper Oxide nanoparticle at concentration of $0.0125 \%$ have the best results in increasing the oil recovery factor by percentage of $24 \%$

- Copper Oxide nanomaterial at concentration of $0.0125 \mathrm{wt} \%$ has the best effect on changing wettability of the reservoir to be strongly water wet.

\section{REFERENCES}

[1] Lake, L.W., et al., Fundamentals of enhanced oil recovery. 2014.

[2] Sheng, J.J., Modern chemical enhanced oil recovery: theory and practice. 2010: Gulf Professional Publishing.

[3] Thomas, S., Enhanced oil recovery-an overview. Oil \& Gas Science and Technology-Revue de l'IFP, 2008. 63(1): p. 9-19. 
[4] Poole Jr, C.P. and F.J. Owens, Introduction to nanotechnology. 2003: John Wiley \& Sons.

[5] Bhushan, B., Springer handbook of nanotechnology. 2017: Springer.

[6] Mokhatab, S., M.A. Fresky, and M.R. Islam, Applications of nanotechnology in oil and gas E\&P. Journal of petroleum technology, 2006. 58(04): p. 48-51.

[7] Peng, B., et al., Applications of nanotechnology in oil and gas industry: Progress and perspective. The Canadian journal of chemical engineering, 2018. 96(1): p. 91-100.

[8] Negin, C., S. Ali, and Q. Xie, Application of nanotechnology for enhancing oil recovery-A review. Petroleum, 2016. 2(4): p. 324-333.

[9] Cheraghian, G. and L. Hendraningrat, A review on applications of nanotechnology in the enhanced oil recovery part B: effects of nanoparticles on flooding. International Nano Letters, 2016. 6(1): p. 1-10.

[10] Cheraghian, G. and L. Hendraningrat, A review on applications of nanotechnology in the enhanced oil recovery part A: effects of nanoparticles on interfacial tension. International Nano Letters, 2016. 6(2): p. 129-138.

[11] He, L., J. Xu, and D. Bin, Application of nanotechnology in petroleum exploration and development. Petroleum Exploration and development, 2016. 43(6): p. 1107-1115.

[12] He, L., et al., Potential application of functional micro-nano structures in petroleum. Petroleum Exploration and Development, 2018. 45(4): p. 745-753.

[13] Ko, S. and C. Huh, Use of nanoparticles for oil production applications. Journal of Petroleum Science and Engineering, 2019. 172: p. 97-114.

[14] Ismail, A., et al., The novel approach for the enhancement of rheological properties of water-based drilling fluids by using multi-walled carbon nanotube, nanosilica and glass beads. Journal of Petroleum Science and Engineering, 2016. 139: p. 264-275.

[15] Aftab, A., et al., Nanoparticles based drilling muds a solution to drill elevated temperature wells: A review. Renewable and Sustainable Energy Reviews, 2017. 76: p. 1301-1313.

[16] Wang, Z., et al., Poly (sodium p-styrene sulfonate) modified Fe3O4 nanoparticles as effective additives in water-based drilling fluids. Journal of Petroleum Science and Engineering, 2018. 165: p. 786-797.

[17] Afolabi, R.O., O.D. Orodu, and I. Seteyeobot, Predictive modelling of the impact of silica nanoparticles on fluid loss of water based drilling mud. Applied Clay Science, 2018. 151: p. 37-45.

[18] Amanullah, M. and M.K. Al-Arfaj, Water-based drilling fluid composition having a multifunctional mud additive for reducing fluid loss during drilling. 2015, Google Patents.

[19] Li, G., J. Zhang, and Y. Hou. Nanotechnology to improve sealing ability of drilling fluids for shale with micro-cracks during drilling. in SPE international oilfield nanotechnology conference and exhibition. 2012. Society of Petroleum Engineers.

[20] Kasiralvalad, E., The great potential of nanomaterials in drilling \& drilling fluid applications. International Journal of Nano Dimension, 2014. 5(5): p. 463-471.

[21] Jain, R., V. Mahto, and V. Sharma, Evaluation of polyacrylamide-grafted-polyethylene glycol/silica nanocomposite as potential additive in water based drilling mud for reactive shale formation. Journal of Natural Gas Science and Engineering, 2015. 26: p. 526-537.

[22] Rana, A., et al., Controlling Shale Swelling and Fluid Loss Properties of Water-Based Drilling Mud via Ultrasonic Impregnated SWCNTs/PVP Nanocomposites. Energy \& Fuels, 2020. 34(8): p. 9515-9523.

[23] Noormohammadi, M. and M. Barmala, Investigating the Effect of Nano Technology on Increasing Utilization in Oil Tanks and Wells in Upstream Industries. International Journal of New Chemistry, 2019. 6(4): p. 289-298.

[24] Kong, X. and M. Ohadi. Applications of micro and nano technologies in the oil and gas industry-overview of the recent progress. in Abu Dhabi international petroleum exhibition and conference. 2010. Society of Petroleum Engineers.

[25] Alencar, M.S.d.M., et al. Nanocoatings Applied to Corrosion Protection at the Oil and Gas Industry Trends. in OTC Brasil. 2013. Offshore Technology Conference.

[26] Roustaei, A., et al. An experimental investigation of polysilicon nanoparticles' recovery efficiencies through changes in interfacial tension and wettability alteration. in SPE international oilfield nanotechnology conference and exhibition. 2012. Society of Petroleum Engineers.

[27] Nowrouzi, I., A.K. Manshad, and A.H. Mohammadi, Effects of concentration and size of TiO2 nanoparticles on the performance of smart water in wettability alteration and oil production under spontaneous imbibition. Journal of Petroleum Science and Engineering, 2019. 183: p. 106357. 
[28] Li, R., et al., Experimental investigation of silica-based nanofluid enhanced oil recovery: the effect of wettability alteration. Energy \& Fuels, 2017. 31(1): p. 188-197.

[29] Moslan, M., et al., Applications of aluminium oxide and zirconium oxide nanoparticles in altering dolomite rock wettability using different dispersing medium. Chemical engineering transactions, 2017. 56: p. 13391344.

[30] Suleimanov, B.A., F. Ismailov, and E. Veliyev, Nanofluid for enhanced oil recovery. Journal of Petroleum Science and Engineering, 2011. 78(2): p. 431-437.

[31] Verga, F., et al., Introducing core-shell technology for conformance control. Oil \& Gas Science and Technology-Revue d'IFP Energies nouvelles, 2017. 72(1): p. 5.

[32] Srinivasan, A. and S.N. Shah. Surfactant-based fluids containing copper-oxide nanoparticles for heavy oil viscosity reduction. in SPE Annual Technical Conference and Exhibition. 2014. Society of Petroleum Engineers.

[33] Ogolo, N., O. Olafuyi, and M. Onyekonwu. Enhanced oil recovery using nanoparticles. in SPE Saudi Arabia section technical symposium and exhibition. 2012. Society of Petroleum Engineers.

Citation: Sayed Gomaa, et.al, "Investigating the Effect of Copper Oxides and Alumina Nanoparticles on Enhanced Oil Recovery in Carbonate Reservoirs", International Journal of Petroleum and Petrochemical Engineering, 6(4), pp. 01-12. DOI: https:// doi.org/10.20431/2454-7980.0604001

Copyright: () 2020 Authors, this is an open-access article distributed under the terms of the Creative Commons Attribution License, which permits unrestricted use, distribution, and reproduction in any medium, provided the original author and source are credited. 